\title{
TEORES DE $\beta$-CAROTENO EM SUPLEMENTOS E BIOMASSA DE Spirulina
}

\author{
Contents of $\beta$-carotene in supplements and biomass of Spirulina
}

\author{
Daniel Barroso de Alencar ${ }^{1}$, Kelma Maria dos Santos Pires-Cavalcante ${ }^{1}$, Jefferson Pablo de Souza Saboya ${ }^{2}$, \\ Márcia Barbosa de Sousa ${ }^{1}$, Wladimir Ronald Lobo Farias ${ }^{2}$, Silvana Saker-Sampaio ${ }^{3}$
}

\begin{abstract}
RESUMO
A cianobactéria Spirulina tem sido utilizada há séculos, tendo em vista suas propriedades nutricionais e medicinais. Neste trabalho, objetivou-se analisar os teores de $\beta$ caroteno tanto em suplementos alimentares a base de Spirulina comercializados em estabelecimentos de produtos naturais no mercado varejista, quanto em S. platensis cultivada em laboratório. Alguns pigmentos carotenóides possuem atividade de vitamina $\mathrm{A}$ e, dentre eles, o $\beta$-caroteno é o que apresenta maior atividade biológica. A extração de $\beta$-caroteno foi feita com metanol:água $(90: 10 \mathrm{v} / \mathrm{v})$, seguida de saponificação e partição em $n$-hexano. As análises cromatográficas foram realizadas em coluna Waters Spherisorb S5 ODS $2(4,6$ × $250 \mathrm{~mm})$, usando metanol:tetrahidrofurano (90:10, v/v) bombeado a $2 \mathrm{~mL} \mathrm{~min}^{-1}$, com registro dos cromatogramas em $450 \mathrm{~nm}$. Os suplementos alimentares a base de Spirulina apresentaram baixos teores de $\beta$-caroteno. A maior concentração foi encontrada em S. platensis cultivada a $24^{\circ} \mathrm{C}$ com fotoperíodo de $16 \mathrm{~h}$ claro e $8 \mathrm{~h}$ escuro. Considerando sua atividade provitamínica, os teores de retinol equivalente (RE) nas microalgas analisadas no presente trabalho foram calculados a partir do $\beta$-caroteno para classificá-las como fonte excelente ou fonte útil de vitamina A.
\end{abstract}

Termos para indexação: Microalga, suplemento alimentar, CLAE.

\begin{abstract}
Spirulina is a cyanobacterium that has been used for several centuries due to its nutritional and medicinal properties. This work has evaluated the contents of $\beta$-carotene both in Spirulina commercialized as food supplement, purchased from natural product shops, and in $S$. platensis reared under laboratory conditions. Some carotenoids exhibit pro-vitamin A activity, and $\beta$-carotene presents the greatest biological activity. $\beta$-Carotene of microalgae was extracted in $90 \%$ aqueous methanol. These extracts were saponified and partitioned in $n$-hexane. Chromatographic analyses were carried out in a Spherisorb column S5 ODS $2(4.6 \times 250 \mathrm{~mm})$, with a mobile phase of methanol:tetrahydrofuran (90:10, v/v) delivered at $2 \mathrm{~mL} \mathrm{~min}^{-1}$ and chromatograms registered at $450 \mathrm{~nm}$ absorbance. Food supplement made with Spirulina presented low content of $\beta$-carotene. The highest content of $\beta$-carotene was detected in $S$. platensis cultivated under $24^{\circ} \mathrm{C}$ with photoperiod of $16 \mathrm{~h} \mathrm{light}$ and $8 \mathrm{~h}$ dark. Considering its pro-vitamin activity, retinol equivalent (RE) was calculated from $\beta$-carotene to determine whether alga material could be claimed as an useful or an excellent source of the vitamins A.
\end{abstract}

Index terms: Microalga, food supplement, HPLC.

(Recebido em 16 de setembro de 2010 e aprovado em 11 de novembro de 2010)

\section{INTRODUÇÃo}

Os povos ocidentais têm usado cianobactérias como parte de sua dieta por séculos. As microalgas comercializadas como suplemento alimentar podem ser coletadas diretamente do ambiente onde elas se desenvolvem naturalmente ou podem ser cultivadas sob condições controladas. Nos últimos anos, houve avanços significativos no cultivo de microalgas cujos reflexos podem ser percebidos em sua aplicação na aquicultura, alimentação humana, produção e extração de pigmentos, fertilizantes, biocombustíveis e metabólitos primários e secundários como toxinas, enzimas e vitaminas (Brennam \& Owende, 2010).
As cianobactérias são capazes de produzir uma vasta diversidade de diferentes compostos biologicamente ativos como mostram as revisões de Abed et al. (2009), Plaza et al. (2009) e Harun et al. (2010). Os efeitos farmacológicos das cianobactérias são utilizados para promovê-las comercialmente (Gantar \& Svircev, 2008), com destaque para a estimulação do sistema imunológico e da microbiota intestinal, atividades antibacteriana, antitumoral, anti-inflamatória, anti-hiperalgésica e antioxidante, eficácia no tratamento de alergias, anemia e ainda sobre os efeitos tóxicos de radiação (Gantar \& Svircev, 2008; Kaushik \& Chauhan, 2008; Shih et al., 2009).

Entre todos os vegetais, a cianofícea Spirulina é a mais rica fonte de ferro e proteína, contendo oito dos dez

1Universidade Federal do Ceará/UFC - Departamento de Engenharia de Pesca/DEP - Laboratório de Bioquímica Marinha/HPLC - Fortaleza, CE ${ }^{2}$ Universidade Federal do Ceará/UFC - Departamento de Engenharia de Pesca/DEP - Laboratório de Planctologia/LABPLANC - Fortaleza, CE ${ }^{3}$ Universidade Federal do Ceará/UFC - Departamento de Engenharia de Pesca/DEP - Laboratório de Bioquímica Marinha/HPLC - Campus do Pici Avenida Mister Hull - s/n - Bloco 827 - Cx P. 6.043 - 60455-970 - Fortaleza, CE - sakersil@gmail.com 
aminoácidos essenciais, usados para combater, respectivamente, a anemia e a má nutrição, prevalentes na população mundial. A presença de outros minerais importantes para o metabolismo humano torna a Spirulina um alimento completo por reunir um grande número de propriedades benéficas, dificilmente encontradas em um único produto natural. Os pigmentos carotenóides estão presentes em quantidades superiores a outras fontes conhecidas na natureza e as ficobilinas podem fortalecer o sistema imunológico, aumentando a resistência do organismo humano contra doenças (Srivastava, 2008). A Spirulina possui uma quantidade mínima de lipídios, constituídos principalmente de ácidos graxos poliinsaturados, como os ácidos $\alpha$-linolênico, $\gamma$-linolênico, linoléico, estearidônico, eicosapentaenóico, docosahexaenóico e araquidônico (Babadzhanov et al., 2004; Ronda \& Lele, 2008; Plaza et al., 2009; Harun et al., 2010).

Com propriedades nutricionais e farmacêuticas, a Spirulina é tratada como um produto nutracêutico (Srivastava, 2008). Entretanto, de acordo com Gantar \& Svircev (2008), a comercialização de biomassa de Spirulina sob a forma desidratada deve ser avaliada quanto à toxicidade e aos efeitos do uso prolongado. Além disso, como as condições de cultivo determinam quais compostos serão produzidos e em que quantidades, é preciso que haja uma regulamentação sobre os produtos comercializados com relação a sua composição para assegurar à satisfação do consumidor.

Conduziu-se este trabalho, com o objetivo de analisar a variação nos teores de $\beta$-caroteno, por sua capacidade de ser convertido em vitamina $\mathrm{A}$, em diferentes marcas de Spirulina comercializadas em cápsulas como suplemento alimentar e determinar as concentrações de $\beta$-caroteno na biomassa de Spirulina platensis cultivada em laboratório em diferentes condições de luminosidade e temperatura.

\section{MATERIAL E MÉTODOS}

\section{Suplemento alimentar à base de Spirulina}

Seis diferentes marcas de suplemento alimentar à base de Spirulina maxima de fabricação nacional e uma de $S$. platensis, cuja matéria-prima foi importada dos Estados Unidos, foram adquiridas em lojas de produtos naturais no mercado varejista de Fortaleza, Ceará, Brasil e analisadas quanto ao teor de $\beta$-caroteno. Os produtos foram denominados A a G, para evitar a identificação dos fabricantes. $\mathrm{O}$ processo de fabricação desses produtos envolve a desidratação da biomassa de Spirulina, acondicionamento em cápsulas com peso individual variando de $250 \mathrm{mg}$ a $400 \mathrm{mg}$ e apresentação em embalagens de 45 ou 90 unidades, dependendo do fabricante.

\section{Spirulina platensis cultivada em laboratório}

A biomassa da microalga $S$. platensis, cultivada no Laboratório de Planctologia do Departamento de Engenharia de Pesca da Universidade Federal do Ceará, foi obtida de cultivos realizados a partir de um mesmo inóculo sob duas condições diferentes de fotoperíodo e temperatura, mantendo-se constante os demais parâmetros. O cultivo denominado $\mathrm{SP}_{24}$ foi realizado com fotoperíodo de 16 horas de claro e 8 horas de escuro e temperatura ambiente de $24 \pm 2^{\circ} \mathrm{C}$. $\mathrm{O}$ outro cultivo, denominado $\mathrm{SP}_{28}$, foi realizado com iluminação constante fornecida por uma lâmpada fluorescente de $40 \mathrm{~W}$ e temperatura ambiente de $28 \pm 2^{\circ} \mathrm{C}$. A biomassa úmida, coletada dos dois cultivos, foi lavada com água destilada para eliminar o sal, congelada a $-21^{\circ} \mathrm{C}$ por 24 horas e, em seguida, liofilizada para obtenção da matéria seca, que foi analisada quanto ao teor de $\beta$-caroteno.

\section{Extração, saponificação e partição dos carotenóides}

Três porções de $1 \mathrm{~g}$ de cada um dos suplementos alimentares comerciais à base de Spirulina e três porções de $0,1 \mathrm{~g}$ de Spirulina cultivada em laboratório $\left(\mathrm{SP}_{24}\right.$ e $\left.\mathrm{SP}_{28}\right)$ foram pesadas separadamente. A extração foi realizada em $10 \mathrm{~mL}$ de metanol:água $(90: 10, \mathrm{v} / \mathrm{v})$. Para a saponificação dos lipídios, o material foi homogeneizado com $5 \%$ de hidróxido de potássio e levado ao banho-maria (Thermomix ${ }^{\circledR} 18 \mathrm{BU}$ ) a $70^{\circ} \mathrm{C}$ por $30 \mathrm{~min}$. Depois de resfriados à temperatura ambiente, os extratos foram centrifugados a $2.000 \times \mathrm{g}$ por $5 \mathrm{~min}$. Após a centrifugação, 1,5 $\mathrm{mL}$ de água Milli-Q e 2,5 mL de $n$-hexano foram adicionados a $5 \mathrm{~mL}$ dos extratos saponificados e deixados por $10 \mathrm{~min}$ em uma plataforma misturadora. Os tubos foram deixados em repouso para permitir a separação das fases. Volumes correspondentes a 1 $\mathrm{mL}$ da fase hexânica foram transferidos para tubos de ensaio, e o solvente evaporado. O resíduo de cada tubo foi então suspenso em $2 \mathrm{~mL}$ de metanol e filtrado em membrana de Chromafil ${ }^{\circledR} \mathrm{CA}-45 / 25 \mathrm{~S}$ de $0,45 \mu \mathrm{m}$, no momento da análise por cromatografia líquida de alta eficiência (CLAE).

\section{Sistema cromatográfico}

O sistema cromatográfico consistiu em uma coluna Waters Spherisorb S5 ODS 2 (4,6 x $250 \mathrm{~mm}$ ) e uma fase móvel constituída de metanol:tetrahidrofurano (90:10, v/v), bombeada a $2 \mathrm{~mL} \mathrm{~min}^{-1}$ usando uma bomba (AKTAbasic 10, modelo P-900, Amersham). Alíquotas de $100 \mu \mathrm{L}$ do resíduo suspenso em metanol foram injetadas manualmente, usando um injetor de amostras Rheodyne 7210 (Hamilton Co.). O monitor (AKTAbasic UV-900) foi ajustado em $450 \mathrm{~nm}$, e os cromatogramas foram registrados por meio do sistema de controle Unicorn $^{\mathrm{TM}}$, versão 5.0. 
Cálculo de $\beta$-caroteno e conversão em retinol equivalente (RE)

Os teores de $\beta$-caroteno nos extratos foram calculados considerando-se a relação entre a quantidade de $\beta$-caroteno injetada na coluna e as áreas dos picos obtidas para o padrão comercial de concentração conhecida e para os extratos das microalgas, usando-se fórmula abaixo. Os resultados foram expressos em $\mu \mathrm{g} \mathrm{g}^{-1}$ peso seco.

$$
\mu g g^{-1}=\frac{\text { área pico extrato microalga }}{\text { área pico } \beta \text {-caroteno padrão }} \times \mu g \quad \beta \text {-caroteno padrão } \times \text { fator de diluição } \times \frac{1 \mathrm{~g}}{\text { peso microalga }}
$$

Os cálculos de RE foram procedidos considerandose que cada $1 \mu \mathrm{g}$ de $\beta$-caroteno corresponde a $0,167 \mu \mathrm{g}$ de RE (Brasil, 2005).

\section{Análise estatística}

Todos os extratos foram preparados em triplicada e cada extrato foi injetado três vezes no cromatógrafo. Os teores de $\beta$-caroteno nos extratos preparados com as diferentes marcas de suplemento alimentar à base de Spirulina foram submetidos à análise de variância unifatorial, seguida do teste de Tukey, para comparação das médias, ambos considerando um nível de significância $(\alpha)=5 \%$. Os teores de $\beta$-caroteno nos extratos preparados com as biomassas de Spirulina cultivadas em laboratório foram comparados por meio do teste $t$ de Student para amostras independentes, também para $\alpha=5 \%$. A comparação dos tempos de retenção do padrão e dos extratos foi feita pelo teste $t$ de Student para dados independentes. Todas as análises foram feitas com auxílio do Programa BioEstat 4.0.

\section{RESULTADOS E DISCUSSÃO}

A quantificação de $\beta$-caroteno nos extratos preparados com os suplementos alimentares à base de Spirulina e com a biomassa de $S$. platensis cultivada em laboratório foi feita com base na curva padrão do $\beta$-caroteno comercial submetido à saponificação e à partição. A existência de correlação $(\mathrm{r}=0,9988, \mathrm{p}<0,05)$ entre a área do pico e a concentração $(10$ a $200 \mu \mathrm{g})$ de $\beta$-caroteno, correspondente a aproximadamente 0,1 a 2,0 $\mu$ g na coluna ( $\mathrm{y}=1,88+90,02 \mathrm{x}, \mathrm{n}=4)$, possibilitou sua quantificação.

No presente trabalho, os tempos de retenção do $\beta$-caroteno padrão $\left(10 \mu \mathrm{g} \mathrm{mL}^{-1}\right)$ e do composto considerado $\beta$-caroteno cromatografado nos extratos das microalgas foram, respectivamente, iguais a 11,32 $\pm 0,32 \mathrm{~min}(\mathrm{n}=14)$ e 11,35 $\pm 0,33 \min (n=47)$, não existindo diferença estatisticamente significativa ( $\alpha=5 \%$ ). A identificação de $\beta$-caroteno nos extratos de Spirulina foi feita com base no tempo de retenção do padrão comercial.

Os teores de $\beta$-caroteno nos extratos dos suplementos alimentares à base de Spirulina foram inferiores àqueles observados nos de $S$. platensis cultivada em laboratório (Tabelas 1 e 2).

Todas as marcas de suplemento alimentar, à base de Spirulina, analisadas no presente trabalho apresentaram $\beta$-caroteno, exceto a marca $\mathrm{G}$. Os resultados foram submetidos à análise de variância unifatorial $(\mathrm{F}=143,24$, $\mathrm{p}<0,0001)$ e ao teste de Tukey. Os teores máximo e mínimo foram encontrados nos extratos da marca $\mathrm{D}\left(14,570 \mu \mathrm{g} \mathrm{g}^{-1}\right.$ peso seco) e $\mathrm{C}\left(1,449 \mu \mathrm{g} \mathrm{g}^{-1}\right.$ peso seco), respectivamente, observando-se uma variação da ordem de dez vezes entre o maior e o menor valor. Tal variação pode estar associada

Tabela 1 - Teores de $\beta$-caroteno (média \pm desvio padrão), nos extratos de diferentes marcas de suplementos alimentares a base de Spirulina comercializados no mercado varejista de Fortaleza, Ceará.

\begin{tabular}{cc}
\hline Suplementos alimentares & $\begin{array}{c}\beta \text {-caroteno } \\
\left(\mu \mathrm{g} \mathrm{g}^{-1} \text { peso seco }\right)\end{array}$ \\
\hline D & $14,570 \pm 0,778^{\mathrm{a}}$ \\
F & $7,614 \pm 0,399^{\mathrm{b}}$ \\
B & $7,494 \pm 0,784^{\mathrm{b}}$ \\
E & $7,387 \pm 1,690^{\mathrm{b}}$ \\
A & $3,572 \pm 0,670^{\mathrm{c}}$ \\
C & $1,449 \pm 0,326^{\mathrm{d}}$ \\
\hline
\end{tabular}

Letras minúsculas iguais indicam inexistência de diferença estatisticamente significativa $(\mathrm{p} \geq 0,05)$ e letras minúsculas diferentes indicam diferença estatisticamente significativa $(p<0,05)$. 
Tabela 2 - Teores de $\beta$-caroteno (média \pm desvio padrão), nos extratos de Spirulina platensis cultivada sob condições controladas em laboratório.

\begin{tabular}{cc}
\hline Extratos & $\begin{array}{c}\beta \text {-caroteno } \\
\left(\mu \mathrm{g} \mathrm{g}^{-1} \text { peso seco }\right)\end{array}$ \\
\hline $\mathrm{SP}_{24}$ & $202,681 \pm 24,626^{\mathrm{a}}$ \\
$\mathrm{SP}_{28}$ & $148,748 \pm 34,746^{\mathrm{b}}$ \\
\hline
\end{tabular}

Letras minúsculas diferentes indicam diferença estatisticamente significativa $(\mathrm{p}=0,0056)$.

ao fato de que a colheita, manipulação e/ou estocagem da matéria-prima, bem como o processo de preparação, também podem interferir na quantidade de carotenóides presentes no material analisado (Rodriguez-Amaya, 2000). Além disso, a composição e a distribuição dos carotenóides nos vegetais são irregulares e afetadas por fatores como estágio do ciclo de vida, clima e/ou condições de produção (Campos \& Rosado, 2005). Por exemplo, a concentração de nitrato no meio de cultura da microalga verde Muriellopsis sp. favorece a acumulação de luteína (Del Campo et al., 2000). $\mathrm{O}$ incremento na produção de $\beta$-caroteno e luteína a partir da microalga verde Dunaliella salina é determinado pelas condições físico-químicas como pH, oxigênio, irradiação e temperatura do cultivo (Lamers et al., 2008).

Neste trabalho, os teores de $\beta$-caroteno encontrados nos extratos preparados com a biomassa de Spirulina cultivada em condições de temperatura e luminosidade diferentes apresentaram diferença estatisticamente significativa: $\mathrm{SP}_{24}\left(202,681 \pm 24,626 \mu \mathrm{g} \mathrm{g}^{-1}\right.$ peso seco) e $\mathrm{SP}_{28}\left(148,748 \pm 34,746 \mu \mathrm{g} \mathrm{g}^{-1}\right.$ peso seco). Como esses fatores físicos influenciam na produção de $\beta$-caroteno, era de se esperar que as microalgas cultivadas em temperaturas mais elevadas e sob iluminação constante a $28^{\circ} \mathrm{C}\left(\mathrm{SP}_{28}\right)$ apresentassem maior teor $\mathrm{de} \beta$-caroteno tendo em vista que sua função nas algas é proteger o aparelho fotossintético contra a fotoxidação e os raios ultravioleta desencadeados por qualquer excesso de luz (Britton et al., 1995; Havaux, 1998). Interessantemente, nessas condições o teor de $\beta$-caroteno correspondeu a $73,4 \%$ daquele registrado no cultivo a $24^{\circ} \mathrm{C}$ com fotoperíodo $\left(\mathrm{SP}_{24}\right)$.

Mais do que um suplemento alimentar saudável, o consumo de Spirulina promete benefícios à saúde do consumidor. Esta microalga tem sido considerada uma valiosa fonte de vitamina A, tendo em vista que, de acordo com Wang et al. (2008), a biodisponibilidade dos carotenos encontrados na Spirulina é muito semelhante àquela de carotenos obtidos de outras fontes como, por exemplo, cenoura e vegetais folhosos, sugerindo que esta microalga apresenta grande potencial como fonte dietária de pró-vitamina A.
De acordo com a quantidade de um determinado nutriente presente em um alimento, ele pode ser classificado como fonte excelente ou fonte útil. Essa classificação considera a participação do referido nutriente na ingestão diária recomendada (IDR). No caso da vitamina A, cuja IDR (Brasil, 2005) é igual a $600 \mu \mathrm{g}$ retinol equivalente (RE), para ser fonte excelente, um alimento deve fornecer $300 \mu \mathrm{g}$ RE ou $50 \%$ da IDR; enquanto para ser fonte útil, ele deve ser responsável por $90 \mu \mathrm{g}$ RE ou $15 \%$ da IDR.

Com base na quantidade de RE $\left(\mu \mathrm{g} \mathrm{g}^{-1}\right)$ estimada a partir do teor de $\beta$-caroteno (Tabela 3), o consumo diário de porções desidratadas de $9,0 \mathrm{~g}$ de $\mathrm{SP}_{24}$ ou $12,6 \mathrm{~g}$ de $\mathrm{SP}_{28}$ forneceriam aproximadamente $50 \%$ da IDR, o que permitiria classificá-las como fontes excelentes de vitamina A. Entretanto, para classificá-las como fontes úteis, as porções seriam reduzidas para $2,7 \mathrm{~g}\left(\mathrm{SP}_{24}\right)$ e $3,9 \mathrm{~g}\left(\mathrm{SP}_{28}\right)$. Para que os suplementos alimentares à base de Spirulina fossem considerados fontes excelentes ou fontes úteis, seria necessário o consumo diário de 123,6 g ou 37,6 g (marca D) e de 1.283,4 g ou 385,0 g (marca C), respectivamente, o que não seria razoável propor a nenhum consumidor. Só para se ter uma ideia, os suplementos alimentares à base de Spirulina são tão pobres com relação ao teor de $\beta$-caroteno, que na melhor das hipóteses seria necessária a ingestão diária de 94 cápsulas de 400 mg para que o produto da marca $\mathrm{D}$ fosse considerado uma fonte útil de vitamina $\mathrm{A}$, quantidade muitas vezes superior à recomendação de consumo dos fabricantes.

$\mathrm{O}$ teor de $\beta$-caroteno em Spirulina fusiformis possibilitou classificá-la como fonte útil ou excelente de vitamina A quando consumidas porções diárias da microalga desidratada de $29 \mathrm{mg}$ ou $96 \mathrm{mg}$, respectivamente (Kandlakunta et al., 2008). A microalga verde Dunaliella salina apresentou $1,4 \mathrm{mg} \beta$-caroteno $\mathrm{g}^{-1}$ alga liofilizada (Denery et al., 2004); nesse caso, o consumo diário de cerca de $2 \mathrm{~g}$ da alga liofilizada seria suficiente para fornecer $50 \%$ da IDR, o que a classificaria como fonte excelente de vitamina A.

Embora existam muitos trabalhos sobre os possíveis efeitos benéficos da Spirulina, outros ainda são necessários para que algumas questões sejam esclarecidas. 
Tabela 3 - Retinol equivalente e quantidade de consumo diário para classificar Spirulina em fonte excelente ou útil de vitamina A.

\begin{tabular}{cccc}
\hline Spirulina & $\begin{array}{c}\text { Retinol equivalente } \\
\left(\mu \mathrm{g} \mathrm{g}^{-1} \text { peso seco) }\right.\end{array}$ & $\begin{array}{c}\text { Porção }(\mathrm{g}) \\
\text { Fonte excelente }\end{array}$ & $\begin{array}{c}\text { Porção }(\mathrm{g}) \\
\text { Fonte útil }\end{array}$ \\
\hline $\mathrm{D}$ & $2,433 \pm 0,130$ & 123,6 & 37,6 \\
$\mathrm{~F}$ & $1,272 \pm 0,067$ & 236,4 & 70,9 \\
$\mathrm{~B}$ & $1,252 \pm 0,131$ & 241,9 & 72,6 \\
$\mathrm{E}$ & $1,234 \pm 0,167$ & 254,5 & 76,4 \\
$\mathrm{~A}$ & $0,597 \pm 0,112$ & 520,4 & 156,1 \\
$\mathrm{C}$ & $0,242 \pm 0,055$ & $1.283,4$ & 385,0 \\
$\mathrm{G}$ & $\mathrm{NQ}$ & - & - \\
$\mathrm{SP}_{24}$ & $33,848 \pm 4,113$ & 9,0 & 2,7 \\
$\mathrm{SP}_{28}$ & $24,841 \pm 5,803$ & 12,6 & 3,9 \\
\hline
\end{tabular}

NQ: Não Quantificado.

Talvez a insuficiência de informações consistentes se deva em grande parte à carência de padrões de qualidade da Spirulina como matéria-prima. Mesmo na China, que é o maior produtor mundial de Spirulina, existe uma preocupação generalizada, sobre a falta de definição de critérios de padronização e garantia da qualidade na produção dessa microalga (Lee, 1997; Li \& Qi, 1997).

\section{CONCLUSÕES}

As variações nos teores de $\beta$-caroteno encontradas nos extratos dos suplementos alimentares à base de Spirulina e naqueles de $S$. platensis cultivada em laboratório confirmam a necessidade de padronização desde o cultivo da microalga até a fabricação desses produtos.

\section{AGRADECIMENTOS}

Ao Conselho Nacional de Desenvolvimento Científico e Tecnológico (CNPq), por concessão de bolsa e apoio financeiro para a realização desta pesquisa, à Fundação Cearense de Apoio ao Desenvolvimento Científico e Tecnológico (FUNCAP) e à Coordenação de Aperfeiçoamento de Pessoal de Nível Superior (CAPES), por concessão de bolsas.

\section{REFERÊNCIAS BIBLIOGRÁFICAS}

ABED, R.M.M.; DOBRETSOV, S.; SUDESH, K. Applications of cyanobacteria in biotechnology. Journal of Applied Microbiology, Malden, v.106, n.1, p.112, Jan. 2009.

BABADZHANOV, A.S.; ABDUSAMATOVA, N.; YUSUPOVA, F.M.; FAIZULLAEVA, N.;
MEZHLUMYAN, L.G.; MALIKOVA, M.K. Chemical composition of Spirulina platensis cultivated in Uzbekistan. Chemistry of Natural Compounds, New York, v.40, n.3, p.276-279, May/June 2004.

BRASIL. Agência Nacional de Vigilância Sanitária. Resolução da Diretoria Colegiada (RDC) no 269, de 22 de setembro de 2005. Brasília, 29 ago. 2005.

BRENNAM, L.; OWENDE, P. Biofuels from microalgae: a review of technologies for production, processing, and extractions of biofuels and co-products. Renewable $\boldsymbol{\&}$ Sustainable Energy Reviews, Oxford, v.14, n.2, p.557577, Feb. 2010.

BRITTON, G.; LIAAEN-JENSEN, S.; PFANDER, H. Carotenoids today and challenges for the future. In: Carotenoids: isolation and analysis.

Switzerland: Birkhauser Verlag, 1995. v.1A, p.13-26.

CAMPOS, F.M.; ROSADO, G.P. Novos fatores de conversão de carotenóides provitamínicos A. Ciências e Tecnologia de Alimentos, Campinas, v.25, n.3, p.571-578, jun./set. 2005.

DEL CAMPO, J.A.; MORENO, J.; RODRÍGUEZ, H.; VARGAS, M.A.; RIVAS, J.; GUERRERO, M.G. Carotenoid content of chlorophycean microalgae: factors determining lutein accumulation in Muriellopsis sp. (Chlorophyta). Journal of Biotechnology, Amsterdam, v.76, n.1, p.51-59, Jan. 2000. 
DENERY, J.R.; DRAGULL, K.; TANG, C.S.; LI, Q.X. Pressurized fluid extraction of carotenoids from Haematococcus pluvialis and Dunaliella salina and kavalactones from Piper methysticum. Analytica Chimica Acta, Amsterdam, v.501, n.2, p.175-181, Jan. 2004.

GANTAR, M.; SVIRCEV, Z. Microalgae and cyanobacteria: food for thought. Journal of Phycology, Oxford, v.44, n.2, p.260-268, Apr. 2008

HARUN, R.; SINGH, M.; FORDE, G.M.; DANQUAH, M.K. Bioprocess engineering of microalgae to produce a variety of consumer products. Renewable and Sustainable Energy Reviews, Oxford, v.14, n.3, p.10371047, Apr. 2010.

HAVAUX, M. Carotenoids as membrane stabilizers in chloroplasts. Trends in Plant Science, Oxford, v.3, n.4, p.147-151, Apr. 1998.

KANDLAKUNTA, B.; RAJENDRA, A.; THINGNGANING, L. Carotene content of some common (cereals, pulses, vegetables, spices and condiments) and unconventional sources of plant origin. Food Chemistry, Oxford, v.106, n.1, p.85-89, Jan. 2008.

KAUSHIK, P.; CHAUHAN, A. In vitro antibacterial activity of laboratory grown culture of Spirulina platensis. Indian Journal of Microbiology, New York, v.48, n.3, p.348-352, Sept. 2008.

LAMERS, P.P.; JANSSEN, M.; DE VOS, R.C.H.; BINO, R.J.; WIJFFELS, R.H. Exploring and exploiting carotenoid accumulation in Dunaliella salina for cellfactory applications. Trends in Biotechnology, London, v.26, n.11, p.631-638, Nov. 2008.
LEE, Y.K. Commercial production of microalgae in the Asia-Pacific rim. Journal of Applied Phycology, Dordrecht, v.9, n.5, p.403-411, 1997.

LI, D.M.; QI, Y.Z. Spirulina industry in China: present status and future prospects. Journal of Applied Phycology, Dordrecht, v.9, n.1, p.25-28, 1997.

PLAZA, M.; HERRERO, M.; CIFUENTES, A.; IBÁÑEZ, E. Innovative natural functional ingredients from microalgae. Journal of Agricultural and Food Chemistry, Washington, v.57, n.16, p.7159-7170, Aug. 2009.

RODRIGUEZ-AMAYA, D.B. Some considerations in generating carotenoid data for food composition tables. Journal of Food Composition and Analysis, San Diego, v.13, n.4, p.641-647, Aug. 2000.

RONDA, S.R.; LELE, S.S. Culture conditions stimulating high $\gamma$-linolenic acid accumulation by Spirulina platensis. Brazilian Journal of Microbiology, São Paulo, v.39, n.4, p.693-697, Oct./Dec. 2008.

SHIH, C.M.; CHENG, S.N.; WONG, C.S.; KUO, Y.L.; CHOU, T.C. Antiinflammatory and antihyperalgesic activity of c-phycocyanin. Anesthesia and Analgesia, Philadelphia, v.108, n.4, p.1303-1310, Apr. 2009.

SRIVASTAVA, P. Neutraceutical Spirulina - I. Vegetos, Meerut, v.21, n.2, p.1-9, Dec. 2008.

WANG, J.; WANG, Y.; WANG, Z.X.; LI, L.; QIN, J.; LAI, W.; FU, Y.; SUTER, P.M.; RUSSELL, R.M.; GRUSAK,

M.A.; TANG, G.W.; YIN, S.A. Vitamin A equivalence of Spirulina $\beta$-carotene in Chinese adults as assessed by using a stable-isotope reference method. American Journal of Clinical Nutrition, Bethesda, v.87, n.6, p.1730-1737, June 2008. 